https://doi.org/10.15407/dopovidi2020.04.025

удК 539.3

\title{
А.М. Багно
}

Институт механики им. С.П. Тимошенко НАН Украины, Киев

E-mail: alexbag2016@gmail.com

\section{О влиянии слоя вязкой сжимаемой жидкости на поверхностную неустойчивость несжимаемого упругого полупространства, подверженного конечным начальным деформациям}

\author{
Представлено академиком НАН Украины А.Н. Гузем
}

Рассматривается задача о распространении гармонических волн в предварительно деформированном упругом несжимаемом полупространстве, взаимодействующем со слоем вязкой сжимаемой жидкости. Исследование проводится на основе трехмерных линеаризованных уравнений теории упругости конечных деформаций для несжимаемого упругого полупространства и трехмерных линеаризованных уравнений Навье-Стокса для слоя вязкой сжимаемой жидкости. Применяются постановка задачи и подход, основанные на использовании представлений общих решений линеаризованных уравнений для упругого тела и жидкости. Получено дисперсионное уравнение, описывающее распространение гармонических волн в гидроупругой системе. Построена дисперсионная кривая поверхностной волны в широком диапазоне частот. Проанализировано влияние конечных начальных деформащий упругого полупространства и толщинь слоя вязкой сжимаемой жидкости на фазовые скорости, коэффициенты затухания, дисперсию поверхностной волны и поверхностную неустойчивость гидроупругого волновода. Числовые результать представлены в виде графиков и дан их анализ.

Ключевые слова: поверхностные волны, фазовая скорость, коэффициент затухания, несжимаемое упругое полупространство, слой вязкой сжимаемой жидкости, конечные начальные деформации, поверхностная неустойчивость.

Линеаризованная теория гидроупругости [1-5], позволяющая учесть свойства, присущие реальным упругим и жидким средам, получила широкое применение как в теоретических фундаментальных, так и в прикладных исследованиях. Использование этой модели дало возможность изучить влияние начальных (остаточных) напряжений в упругом теле, а также вязкость и сжимаемость жидкости на закономерности распространения волн в гидроупругих системах. Являясь более общей моделью, она позволяет исследовать не только волноводные свойства, а также возможность возникновения в гидроупругих системах поверхностной неустойчивости.

Ци тування: Багно А.М. О влиянии слоя вязкой сжимаемой жидкости на поверхностную неустойчивость несжимаемого упругого полупространства, подверженного конечным начальным деформациям Допов. Наи. акад. наук Укр. 2020. № 4. С. 25-33. https://doi.org/10.15407/dopovidi2020.04.025 
В данной работе для исследования влияния слоя вязкой жидкости на поверхностную неустойчивость упругого полупространства применяются модели, учитывающие начальные напряжения в твердом теле, а также вязкость и сжимаемость жидкой среды. При исследовании используются трехмерные линеаризованные уравнения Навье-Стокса для жидкости и трехмерные линеаризованные уравнения теории упругости конечных деформаций для твердого тела. При этом предполагается, что жидкость является ньютоновской, находится в состоянии покоя и тепловые эффекты не учитываются. В качестве подхода выбраны постановки задач и метод, основанные на применении представлений общих решений линеаризованных уравнений движения вязкой сжимаемой жидкости и предварительно напряженного несжимаемого упругого тела, предложенные в работах [1-5].

Постановка задачи. В работе рассмотрим такие динамические процессы в гидроупругой системе, при которых возникающие дополнительные деформации, т.е. возмущения деформаций значительно меньше начальных. Исследуем гармонические волновые процессы малой амплитуды.

Предположим, что изотропное нелинейно-упругое твердое тело, упругий потенциал которого является произвольной дважды непрерывно-дифференцируемой функцией компонент тензора деформаций Грина, занимает объем: $-\infty<z_{1}<\infty,-\infty<z_{2} \leqslant 0,-\infty<z_{3}<\infty$ и контактирует со слоем вязкой сжимаемой жидкости, заполняющей объем: $-\infty<z_{1}<\infty$, $0 \leqslant z_{2} \leqslant h,-\infty<z_{3}<\infty$. Примем, что внешние силы, действующие на указанные среды, распределены равномерно вдоль оси $O z_{3}$. В этом случае задача является плоской и можно ограничиться изучением процесса распространения волн в плоскости $O z_{1} z_{2}$. Следовательно, указанная задача сводится к решению системы линеаризованных уравнений гидроупругости при следующих граничных условиях:

$$
\begin{aligned}
& \left.\tilde{Q}_{1}\right|_{z_{2}=0}=\left.\tilde{P}_{1}\right|_{z_{2}=0} ;\left.\quad \tilde{Q}_{2}\right|_{z_{2}=0}=\left.\tilde{P}_{2}\right|_{z_{2}=0} ;\left.\tilde{P}_{1}\right|_{z_{2}=h}=0 ;\left.\tilde{P}_{2}\right|_{z_{2}=h}=0 ; \\
& \left.v_{1}\right|_{z_{2}=0}=\left.\frac{\partial u_{1}}{\partial t}\right|_{z_{2}=0} ;\left.\quad v_{2}\right|_{z_{2}=0}=\left.\frac{\partial u_{2}}{\partial t}\right|_{z_{2}=0} .
\end{aligned}
$$

Воспользуемся постановками задач гидроупругости для тел с начальными напряжениями и вязкой жидкости, а также представлениями общих решений, предложенными в работах [1-5]. В дальнейшем исследуем волновые процессы в предварительно деформированных несжимаемых упругих телах, взаимодействующих с вязкой жидкостью, начальное состояние которых является однородным. В случае однородного напряженно-деформированного состояния для плоского случая общие решения имеют вид [1-5]

$$
\begin{aligned}
& u_{1}=-\frac{\partial^{2} \chi_{1}}{\partial z_{1} \partial z_{2}} ; u_{2}=\lambda_{1} q_{1} \lambda_{2}^{-1} q_{2}^{-1} \frac{\partial^{2}}{\partial z_{1}^{2}} \chi_{1} \\
& p=\lambda_{1}^{-1} q_{1}^{-1}\left\{\lambda_{1}^{2}\left[\lambda_{1}^{2} a_{11}+s_{11}^{0}-\lambda_{1} \lambda_{2} q_{1} q_{2}^{-1}\left(a_{12}+\mu_{12}\right)\right] \frac{\partial^{2}}{\partial z_{1}^{2}}+\lambda_{2}^{2}\left(\lambda_{1}^{2} \mu_{12}+s_{22}^{0}\right) \frac{\partial^{2}}{\partial z_{2}^{2}}-\rho \frac{\partial^{2}}{\partial t^{2}}\right\} \frac{\partial}{\partial z_{2}} \chi_{1} \\
& v_{1}=\frac{\partial^{2} \chi_{2}}{\partial z_{1} \partial t}+\frac{\partial^{2} \chi_{3}}{\partial z_{2} \partial t} ; v_{2}=\frac{\partial^{2} \chi_{2}}{\partial z_{2} \partial t}-\frac{\partial^{2} \chi_{3}}{\partial z_{1} \partial t}
\end{aligned}
$$


где введенные функции $\chi_{i}$ являются решениями следующих уравнений:

$$
\begin{aligned}
& {\left[\frac{\partial^{4}}{\partial z_{1}^{4}}+\frac{\lambda_{2}^{4} q_{2}^{2}\left(\lambda_{1}^{2} \mu_{12}+s_{22}^{0}\right)}{\lambda_{1}^{4} q_{1}^{2}\left(\lambda_{2}^{2} \mu_{12}+s_{11}^{0}\right)} \frac{\partial^{4}}{\partial z_{2}^{4}}-\frac{\rho}{\lambda_{1}^{2}\left(\lambda_{2}^{2} \mu_{12}+s_{11}^{0}\right)} \frac{\partial^{4}}{\partial z_{1}^{2} \partial t^{2}}+\right.} \\
& +\frac{q_{1} q_{2}^{-1}\left(\lambda_{2}^{2} a_{22}+s_{22}^{0}\right)+q_{1}^{-1} q_{2}\left(\lambda_{1}^{2} a_{11}+s_{11}^{0}\right)-2 \lambda_{1} \lambda_{2}\left(a_{12}+\mu_{12}\right)}{\lambda_{1}^{2} \lambda_{2}^{-2}\left(\lambda_{2}^{2} \mu_{12}+s_{11}^{0}\right) q_{1} q_{2}^{-1}} \frac{\partial^{4}}{\partial z_{1}^{2} \partial z_{2}^{2}}- \\
& \left.-\frac{\lambda_{2}^{2} q_{2}^{2} \rho}{\lambda_{1}^{4} q_{1}^{2}\left(\lambda_{2}^{2} \mu_{12}+s_{11}^{0}\right)} \frac{\partial^{4}}{\partial z_{2}^{2} \partial t^{2}}\right] \chi_{1}=0 ; q_{i}=\lambda_{i}^{-1} ; \lambda_{1} \lambda_{2}=1 ; \\
& {\left[\left(1+\frac{4 v^{*}}{3 a_{0}^{2}} \frac{\partial}{\partial t}\right)\left(\frac{\partial^{2}}{\partial z_{1}^{2}}+\frac{\partial^{2}}{\partial z_{2}^{2}}\right)-\frac{1}{a_{0}^{2}} \frac{\partial^{2}}{\partial t^{2}}\right] \chi_{2}=0 ;\left[\frac{\partial}{\partial t}-v^{*}\left(\frac{\partial^{2}}{\partial z_{1}^{2}}+\frac{\partial^{2}}{\partial z_{2}^{2}}\right)\right] \chi_{3}=0 .}
\end{aligned}
$$

Выше приняты следующие обозначения: $\tilde{Q}_{i}$ и $\tilde{P}_{i}-$ составляющие напряжений, соответственно, в упругом теле и жидкости; $u_{i}$ - компоненты вектора смещений упругого тела $\mathbf{u} ; v_{i}-$ составляющие вектора возмущений скорости жидкости $\boldsymbol{v}$ относительно состояния покоя; $\lambda_{i}-$ удлинения упругого тела в направлениях координатных осей; $a_{i j}, \mu_{i j}-$ величины, определяемые из уравнений состояния и зависящие от вида упругого потенциала $[1,2$, $6,7] ; s_{i i}^{0}-$ начальные напряжения; $\rho-$ плотность материала упругого полупространства; $\rho_{0}$ и $a_{0}-$ плотность и скорость звука в жидкости в состоянии покоя; $v^{*}$ и $\mu^{*}-$ кинематический и динамический коэффициенты вязкости жидкости.

Для анализа распространения возмущений, гармонически изменяющихся во времени, решения системы уравнений определяем в классе бегущих волн

$$
\chi_{j}=X_{j}\left(z_{2}\right) \exp \left[i\left(k z_{1}-\omega t\right)\right](j=\overline{1,3}),
$$

где $k(k=\beta+i \gamma)-$ волновое число; $\gamma-$ коэффициент затухания волны; $\omega-$ круговая частота; $i$ - мнимая единица $(i=\sqrt{-1})$.

Заметим, что выбранный в работе класс гармонических волн, являясь наиболее простым и удобным в теоретических исследованиях, не ограничивает общности полученных результатов, поскольку линейная волна произвольной формы, как известно, может быть представлена набором гармонических составляющих. Далее применяя метод Фурье, приходим к трем задачам о собственных значениях для уравнений движения упругого тела и жидкости. Решая их, определяем собственные значения и соответствующие собственные функции. После подстановки полученных общих решений в граничные условия (1), (2) получаем однородную систему линейных алгебраических уравнений относительно произвольных постоянных. Исходя из условия существования нетривиального решения этой системы, получаем дисперсионное уравнение

$$
\operatorname{det}\left\|e_{l m}\left(c, \gamma, \mu, \rho, \lambda_{i}, a_{i j}, \mu_{i j}, s_{i i}^{0}, \rho_{0}, a_{0}, \mu^{*}, \omega h / c_{s}\right)\right\|=0 \quad(l, m=\overline{1,6}),
$$

где $c-$ фазовая скорость волн в гидроупругой системе; $h-$ толщина жидкого слоя; $c_{s}-$ скорость волны сдвига в материале упругого тела $\left(c_{s}^{2}=\mu / \rho\right) ; \mu-$ модуль сдвига материала упругого тела. 


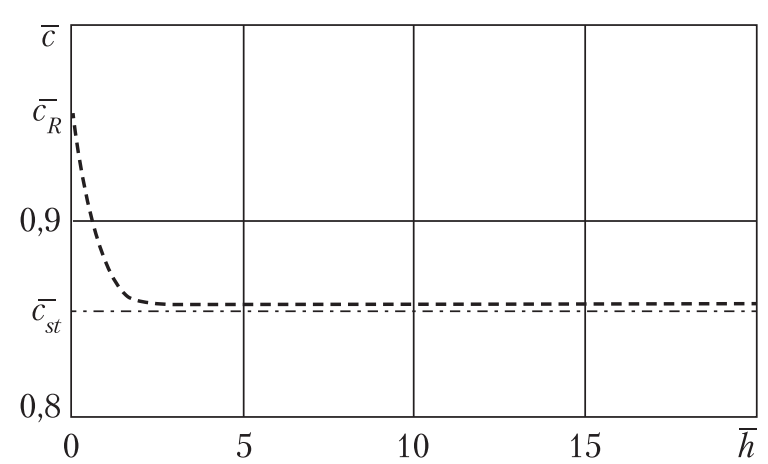

Puc. 1

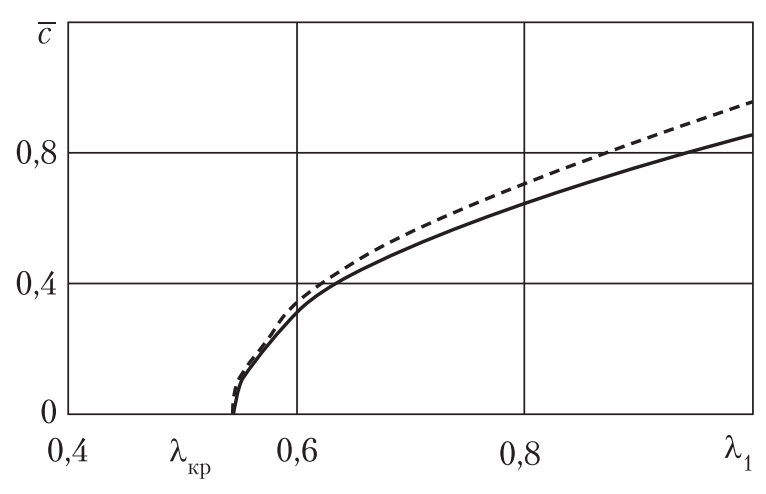

Puc. 2

Отметим, что полученное дисперсионное уравнение (3) не зависит от формы упругого потенциала. Оно является наиболее общим и из него можно получить соотношения для ряда частных случаев, которые рассмотрены в работах $[1,2,8-10]$. В частности, если $a_{0}$ устремить к бесконечности, то (3) переходит в уравнение для определения параметров мод в случае взаимодействия упругого полупространства со слоем несжимаемой вязкой жидкости. При $\rho_{0}=0$ равенство (3) перейдет в уравнение для определения скоростей волн Рэлея $[1,2,9]$. В случае если $\rho_{0} \neq 0$ и $h \rightarrow \infty$, то равенство перейдет в соотношение, определяющее фазовую скорость волны Стоунли $[1,2,10]$.

Указанные частные случаи учитывают наличие начальных деформаций в упругом полупространстве. Модели, основанные на различных вариантах теории малых начальных деформаций, на уравнениях приближенных прикладных двумерных теорий как для предварительно напряженных, так и для тел без начальных деформаций, на линейных соотношениях классической теории упругости, являются частными случаями, рассматриваемого в работе, и следуют из него при введении дополнительных упрощающих предположений. Задачи, которые были рассмотрены в рамках модели, учитывающей начальные напряжения, приведены в $[1,2,5,6,8-10]$. Если положить $s_{i i}^{0}=0 \quad\left(\lambda_{i}=1\right)$ и $\mu^{*}=0$, то получим равенства для основательно исследованных в рамках классической теории гидроупругости волн Рэлея и Стоунли-Шольте [11].

Числовые результаты. В дальнейшем дисперсионное уравнение (3) решаем численно. При этом расчеты проводим для гидроупругой системы, материалы составляющих которой являются реальными. В качестве материала для упругого полупространства выбираем высокоэластичную несжимаемую резину, упругие свойства которой описываются упругим потенциалом Трелоара. При этом механические параметры гидроупругой системы выбираем следующими: упругое полупространство - $\rho=1200$ кг $/ \mathrm{m}^{3}, \mu=1,2 \cdot 10^{6}$ Па; слой жидкости $-\rho_{0}=1000$ кг $/ \mathrm{M}^{3}, a_{0}=1459,5 \mathrm{~m} / \mathrm{c}, \bar{\mu}^{*}=0,001, \bar{a}_{0}=a_{0} / c_{s}=46,153442$. У этого волновода материал упругого тела (резина) является податливым и мягким. Кроме того, при решении предполагалось, что начальное напряженное состояние удовлетворяло соотношениям $s_{11}^{0} \neq 0, s_{22}^{0}=0$. Как показано в работе [7], при такой загрузке нет аналогии между задачами в линеаризованной и линейной постановках. Поэтому результаты для тел с начальными напряжениями не могут быть получены из решений соответствующих линейных задач. 


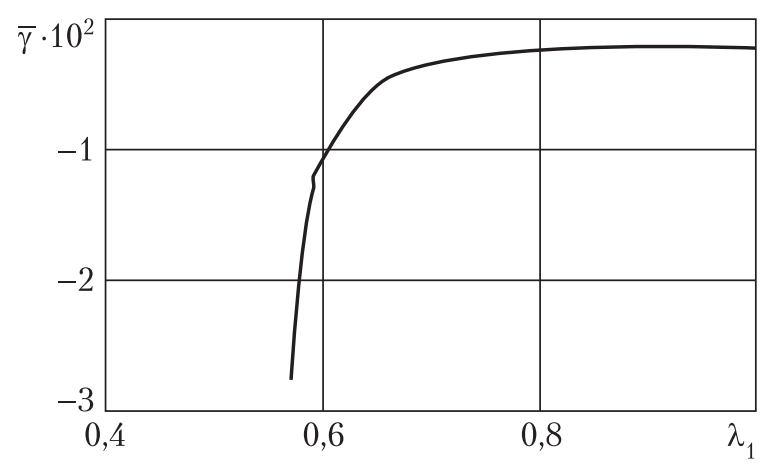

Puc. 3

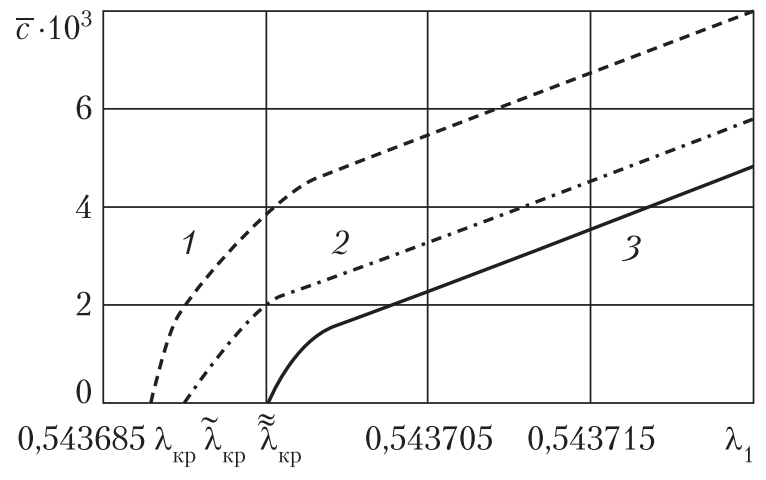

Puc. 4

Заметим, что уравнение (3) выведено без каких-либо дополнительных требований к виду функции упругого потенциала, поэтому оно имеет место для упругих потенциалов произвольной формы. Далее дисперсионное уравнение (3) решаем численно.

Результаты вычислений в виде графиков представлены на рис. 1-4. На рис. 1 приведен график дисперсионной кривой для гидроупругого волновода, отражающий зависимость безразмерной величины фазовой скорости волны $\bar{c}\left(\bar{c}=c / c_{s}\right)$ от безразмерной величины толщины слоя вязкой $\left(\bar{\mu}^{*}=0,001\right)$ жидкости (частоты) $\bar{h}\left(\bar{h}=\omega h / c_{s}\right)$ при отсутствии начальных напряжений $\left(\lambda_{1}=1\right)$. На этом рисунке штрихпунктирной линией обозначена асимптотика, к которой стремится фазовая скорость $\bar{c}$ этой волны при возрастании частоты $\bar{h}(\bar{h} \rightarrow \infty)$.

Характер влияния предварительного деформирования упругого полупространства на скорости поверхностных волн иллюстрируют графики на рис. 2, на котором представлены зависимости величин фазовых скоростей $\bar{c}$ этих мод от изменения величины $\lambda_{1}$. Этот рисунок содержит результаты вычислений для предварительно сжатого $\left(\lambda_{1} \leqslant 1\right)$ упругого полупространства. При этом график, изображенный сплошной линией, получен для гидроупругой системы, толщина вязкого $\left(\bar{\mu}^{*}=0,001\right)$ жидкого слоя $\bar{h}$ которой равна 20 , а штриховая линия отвечает упругому полупространству, не взаимодействующему с жидкостью $\left(\bar{\rho}_{0}=0\right)$.

На рис. 3 приведен график зависимости безразмерной величины коэффициента затухания поверхностной волны $\bar{\gamma}\left(\bar{\gamma}=\gamma / k_{s}, k_{s}-\right.$ волновое число волны сдвига в материале упругого полупространства) от величины $\lambda_{1}$ для гидроупругой системы $\left(\bar{\mu}^{*}=0,001\right)$, упругое полупространство которой подвержено начальному сжатию $\left(\lambda_{1} \leqslant 1\right)$. Как и в предыдущем случае, этот график получен для гидроупругой системы, толщина вязкого $\left(\bar{\mu}^{*}=0,001\right)$ жидкого слоя $\bar{h}$ которой равна 20.

Графики на рис. 4 представляют значения фазовых скоростей поверхностных волн для сильно сжатого упругого полупространства $\left(0,543685 \leqslant \lambda_{1} \leqslant 0,543725\right)$.

Приведенный на рис. 4 график, изображенный сплошной линией 3, получен для гидроупругой системы, толщина вязкого $\left(\bar{\mu}^{*}=0,001\right)$ жидкого слоя $\bar{h} 20$. На этом рисунке штриховая линия 1 отвечает упругому полупространству, не взаимодействующему с жидкостью $\left(\bar{\rho}_{0}=0\right)$. Характер влияния предварительного сжатия $\left(0,543685<\lambda_{1} \leqslant 0,543725\right)$ упругого полупространства на величину фазовой скорости $\bar{c}$ поверхностной волны в гидроупругой 
системе со слоем идеальной жидкости $\left(\bar{\mu}^{*}=0\right)$ иллюстрирует график, представленный штрихпунктирной линией 2.

Анализ числовых результатов. Из графика, представленного на рис. 1, следует, что в гидроупругой системе, компонентами которой является слой вязкой $\left(\bar{\mu}^{*}=0,001\right)$ жидкости $\left(\bar{a}_{0}=46,1534424\right)$ и упругое полупространство из податливого материала, распространяется лишь одна волна. Величина ее фазовой скорости изменяется от скорости волны Рэлея $\bar{c}_{R}\left(\bar{c}_{R}=c_{R} / c_{s}=0,9553125\right.$ для $\left.\lambda_{1}=1\right)$ при $\bar{h} \rightarrow 0$ до скорости волны Стоунли $\bar{c}_{s t}$ $\left(\bar{c}_{s t}=c_{s t} / c_{s}=0,8579379\right.$ для $\left.\lambda_{1}=1\right)$ при $\bar{h} \rightarrow \infty$. В рассматриваемом случае механические параметры гидроупругой системы резина - вода таковы, что скорость распространения звуковой волны в жидкости $\bar{a}_{0}\left(\bar{a}_{0}=46,153442\right)$ больше скорости рэлеевской волны $\bar{c}_{R}$ $\left(\bar{c}_{R}=0,9553125\right.$ для $\left.\lambda_{1}=1\right)$. Согласно результатам, полученным в работе [12], при таком соотношении между величинами скоростей волн в данной гидроупругой системе в коротковолновой части спектра поверхностная мода, распространяясь вдоль границы раздела сред, локализуется, преимущественно, в приконтактной области упругого полупространства.

Кроме того, как видно из графиков, представленных на рис. 2 и 4, сжатие упругого полупространства $\left(\lambda_{1}<1\right)$ приводит к уменьшению величин фазовых скоростей $\bar{c}$ поверхностных волн.

Поверхностная неустойчивость гидроупругой системы. Графики на рис. 2 и 4 для чисто упругого полупространства, изображенные штриховыми линиями 1, показывают, что при сжатии и $\lambda_{1} \approx 0,54$ (более точное значение $\lambda_{1} \approx 0,543688$ ), то есть при уменьшении длины высокоэластичного несжимаемого тела на 46 \% величина фазовой скорости волны Рэлея $\bar{c}_{R}$ обращается в нуль. Это свидетельствует о том, что в условиях плоского напряженно-деформированного начального состояния для высокоэластичного несжимаемого неогуковского тела при сжатии $\lambda_{1} \approx 0,54$ возникает явление поверхностной неустойчивости упругого полупространства. Отметим, что это значение совпадает с величиной, ранее полученной в теории устойчивости [7] и соответствует значению параметра критического укорочения $\lambda_{\text {кр. }}$.

Из графиков (сплошная линия 3 на рис. 4) следует, что в гидроупругом волноводе фазовая скорость поверхностной волны Стоунли $\bar{c}_{s t}$ обращается в нуль при $\lambda_{1} \approx 0,543695$. Это свидетельствует о том, что в условиях плоского напряженно-деформированного начального состояния поверхность упругого полупространства гидроупругой системы, контактирующая со слоем вязкой жидкости, при сжатии $\tilde{\tilde{\lambda}}_{\text {кр }}=\lambda_{1} \approx 0,543695$ теряет поверхностную устойчивость. Для чисто упругого полупространства, как отмечено ранее, явление поверхностной неустойчивости возникает при $\lambda_{\text {кр }}=\lambda_{1} \approx 0,543688$. Эти различия между $\tilde{\tilde{\lambda}}_{\text {кр }}$ и $\lambda_{\text {кр }}$ свидетельствуют о том, что наличие слоя вязкой сжимаемой жидкости $\left(\bar{\mu}^{*}=0,001\right)$ приводит к понижению порога поверхностной неустойчивости гидроупругого волновода и возникновению ее раньше $\left(\tilde{\tilde{\lambda}}_{\text {кр }}>\lambda_{\text {кр }}\right)$ при меньшем сжатии $\tilde{\tilde{\lambda}}_{\text {кр }}=\lambda_{1} \approx 0,543695>\lambda_{\text {кр }}=$ $=\lambda_{1} \approx 0,543688$ ). Из графиков, приведенных на рис. 4, также следует, что в гидроупругой системе, состоящей из упругого полупространства и слоя идеальной $\left(\bar{\mu}^{*}=0\right)$ жидкости (штрихпунктирная линия 2), потеря поверхностной устойчивости $\left(\tilde{\lambda}_{\text {кр }}=\lambda_{1} \approx 0,54369\right.$ ) возникает раньше [13], чем в чисто упругом полупространстве $\left(\bar{\rho}_{0}=0\right)$, но позже, чем в гидроупругой системе с вязким жидким слоем $\left(\bar{\mu}^{*}=0,001\right)$. При этом для значений пара- 
метров критического укорочения имеет место соотношение $\tilde{\tilde{\lambda}}_{\text {кр }}=\lambda_{1} \approx 0,543695>\tilde{\lambda}_{\text {кр }}=$ $=\lambda_{1} \approx 0,54369>\lambda_{\text {кр }}=\lambda_{1} \approx 0,543688$.

Таким образом, развитая линеаризованная теория волн применительно к высокоэластичным несжимаемым телам позволяет исследовать волновые процессы не только в общем и ряде частных случаев, но также возможность и условия возникновения явления поверхностной неустойчивости как в упругом полупространстве, так и в гидроупругих системах.

Выводы. Анализ полученных числовых результатов показал, что влияние конечных начальных деформаций упругого полупространства на характеристики волнового процесса в гидроупругой системе проявляется не только количественно, но и качественно. Большие предварительные деформации могут привести не только к изменению величин фазовых скоростей и дисперсионных свойств поверхностных волн, но и к более существенному изменению параметров волнового процесса в гидроупругой системе в целом. В результате их действия в упруго-жидкостном волноводе может возникнуть явление поверхностной неустойчивости, приводящее к прекращению процесса распространения волн и переноса волновой энергии.

\section{ЦИТИРОВАННАЯ ЛИТЕРАТУРА}

1. Гузь А.Н. Упругие волны в телах с начальными (остаточными) напряжениями: 2 части. Saarbrucken: LAMBERT Acad. Publ., 2016.

2. Гузь А.Н. Динамика сжимаемой вязкой жидкости. Киев: А.С.К., 1998. 350 с.

3. Guz A.N. Dynamics of compressible viscous fluid. Cambridge: Cambridge Sci. Publ., 2009. 428 p.

4. Гузь А.Н. Введение в динамику сжимаемой вязкой жидкости. Saarbrucken: LAMBERT Acad. Publ. RU, 2017. $244 \mathrm{c}$.

5. Guz A. N. Aerohydroelasticity problems for bodies with initial stresses. Int. Appl. Mech. 1980. 16, № 3. P. $175-190$.

6. Гузь А.Н., Жук А.П., Махорт Ф.Г. Волны в слое с начальными напряжениями. Киев: Наук. думка, 1976. $104 \mathrm{c}$.

7. Гузь А.Н. Устойчивость упругих тел при конечных деформациях. Киев: Наук. думка, 1973. 272 с.

8. Guz A. N., Zhuk A. P., Bagno A. M. Dynamics of elastic bodies, solid particles, and fluid parcels in a compressible viscous fluid (review). Int. Appl. Mech. 2016. 52, № 5. P. 449-507.

9. Babich S.Y., Guz A.N., Zhuk A.P. Elastic waves in bodies with initial stresses. Int. Appl. Mech. 1979. 15 , № 4. P. 277-291.

10. Жук А.П. Волны Стонли в среде с начальными напряжениями. Прикл. механика. 1980. 16 , № 1. C. $113-116$.

11. Викторов И.А. Звуковые поверхностные волны в твердых телах. Москва: Наука, 1981. 288 с.

12. Гузь А.Н., Багно А.М. Об акустических волнах в слое вязкой жидкости, взаимодействующем с упругим полупространством. Допов. Нащ. акад. наук Укр. 2018. № 6. С. 40-48. https://doi.org/10.15407/ dopovidi2018.06.040

13. Багно А.М. О влиянии слоя идеальной сжимаемой жидкости на поверхностную неустойчивость несжимаемого упругого полупространства, подверженного конечным начальным деформациям. Допов. Наи. акад. наук Укр. 2020. № 1. С. 24-32. https://doi.org/10.15407/dopovidi2020.01.024

Поступило в редакцию 21.03.2019

\section{REFERENCES}

1. Guz, A. N. (2016). Elastic waves in bodies with initial (residual) stresses. 2 parts. Saarbrucken: LAMBERT Acad. Publ. (in Russian).

2. Guz, A. N. (1998). Dynamics of compressible viscous fluid. Kiev: A.C.K. (in Russian). 
3. Guz, A. N. (2009). Dynamics of compressible viscous fluid. Cambridge: Cambridge Sci. Publ.

4. Guz, A. N. (2017). Introduction to dynamics of compressible viscous fluid. Saarbrucken: LAMBERT Acad. Publ. RU (in Russian).

5. Guz, A. N. (1980). Aerohydroelasticity problems for bodies with initial stresses. Int. Appl. Mech., 16, No. 3, pp. 175-190. https//doi.org/10.1007/BF00885084

6. Guz, A.N., Zhuk, A.P. \& Makhort, F.G. (1976). Waves in layer with initial stresses. Kiev: Naukova Dumka (in Russian).

7. Guz, A. N. (1973). Stability of elastic bodies under finite deformations. Kiev: Naukova Dumka (in Russian).

8. Guz, A. N., Zhuk, A. P. \& Bagno, A. M. (2016). Dynamics of elastic bodies, solid particles, and fluid parcels in a compressible viscous fluid (review). Int. Appl. Mech., 52, No. 5, pp. 449-507. https//doi.org/10.1007/ S10778-016-0770-6

9. Babich, S. Y., Guz, A. N. \& Zhuk, A. P. (1979). Elastic waves in bodies with initial stresses. Int. Appl. Mech., 15, No. 4, pp. 277-291. https//doi.org/10.1007/BF00884760

10. Zhuk, A. P. (1980). Stoneley wave in a medium with initial stresses. J. Appl. Mech., 16, No. 1, pp. 113-116 (in Russian).

11. Viktorov, I. A. (1981). Sound surface waves in solids. Moscow: Nauka (in Russian).

12. Guz, A. N. \& Bagno, A. M. (2018). On the acoustic waves in a layer of a viscous fluid interacting with the elastichalf-space. Dopov.Nac.acad.nauk Ukr.,No.6,pp.40-48.https://doi.org/10.15407/dopovidi2018.06.040 (in Russian).

13. Bagno, A. M. (2020). On the influence of layer of an ideal compressible fluid on the surface instability of incompressible elastic half-space exposed to the finite initial deformations. Dopov. Nac. acad. nauk Ukr., No. 1, pp. 24-32. https://doi.org/10.15407/dopovidi2020.01.024

Received 21.03.2019

\section{О.М. Багно}

Інститут механіки ім. С.П. Тимошенка НАН України, Київ

E-mail: alexbag2016@gmail.com

\section{ПРО ВПЛИВ ШАРУ В’ЯЗКОЇ СТИСЛИВОЇ РІДИНИ \\ НА ПОВЕРХНЕВУ НЕСТІЙКІСТЬ НЕСТИСЛИВОГО ПРУЖНОГО ПІВПРОСТОРУ, ПІДДАНОГО \\ СКІНЧЕННИМ ПОЧАТКОВИМ ДЕФОРМАЦІЯМ}

Розглядається задача про поширення гармонічних хвиль у попередньо деформованому нестисливому півпросторі, що взаємодіє з шаром в'язкої стисливої рідини. Дослідження проведено на основі тривимірних лінеаризованих рівнянь теорії пружності скінченних деформацій для нестисливого пружного півпростору та тривимірних лінеаризованих рівнянь Нав'є-Стокса для шару в'язкої стисливої рідини. Застосовано постановку задачі та підхід, засновані на використанні представлень загальних розв'язків лінеаризованих рівнянь для пружного тіла та рідини. Отримано дисперсійне рівняння, яке описує поширення гармонічних хвиль у гідропружній системі. Побудована дисперсійна крива поверхневої хвилі в широкому діапазоні частот. Проаналізовано вплив скінченних початкових деформацій пружного півпростору та товщини шару в'язкої стисливої рідини на фазові швидкості, коефіцієнти згасання, дисперсію поверхневої хвилі та поверхневу нестійкість гідропружного хвилеводу. Числові результати наведено у вигляді графіків і дано їх аналіз.

Ключові слова: поверхневі хвилі, фазова швидкість, коефіцієнт згасання, нестисливий пружний півпростір, шар в'язкої стисливої рідини, скінченні початкові деформації, поверхнева нестійкість. 


\section{A.M. Bagno}

S.P. Timoshenko Institute of Mechanics of the NAS of Ukraine, Kyiv E-mail: alexbag2016@gmail.com

\section{ON THE INFLUENCE OF A LAYER OF A VISCOUS COMPRESSIBLE FLUID ON THE SURFACE INSTABILITY OF THE INCOMPRESSIBLE ELASTIC HALF-SPACE EXPOSED TO FINITE INITIAL DEFORMATIONS}

The problem of propagation of harmonic waves in the pre-deformed incompressible elastic half-space that interacts with a layer of an viscous compressible fluid is considered. The study is based on the three-dimensional linearized equations of theory of elasticity of finite deformations for the incompressible elastic half-space and the three-dimensional linearized Navier-Stokes equations for a layer of a viscous compressible fluid. The approach based on the utilization of representations of general solutions of the linearized equations for an elastic solid and a fluid is applied. A dispersion equation, which describes propagation of harmonic waves in a hydroelastic system, is obtained. The dispersion curve for surface waves over a wide range of frequencies is constructed. The effects of finite initial deformations of the elastic half-space and of the thickness of the layer of a viscous compressible fluid on the phase velocities, attenuation coefficients, dispersion of the surface waves, and surface instability of a hydroelastic waveguide are analyzed. The numerical results are presented in the form of graphs, and their analysis is given.

Keywords: surface waves, phase velocity, attenuation coefficient, incompressible elastic half-space, layer of viscous compressible fluid, finite initial deformations, surface instability. 